\title{
Low-Loss Energy-Filtered Transmission Electron Microscopy for Imaging and Analysis of Nanoparticles
}

\author{
A.P. Merkle*, S. Pokrant**, S. Irsen*** \\ * $\quad$ Carl Zeiss SMT Inc. Peabody, MA USA \\ ** Carl Zeiss NTS GmbH, Oberkochen, Germany \\ *** Forschungszentrum Caesar, 53175 Bonn, Germany
}

Atomic structure and composition are key components in assembling a working understanding of the catalytic, optical and other properties of nanoparticles, particularly in the fields of nanophotonics and optoelectronics. While conventional transmission electron microscopy (TEM) is well-suited to characterize such materials with sufficient spatial resolution, the combination of energy loss spectroscopy (EELS) techniques with imaging has enabled researchers to complement high spatial resolution information with local chemical and elemental information. The so called "data cube" built up from the spatial dimensions of the image and by the energy dimension of the spectrum can be acquired in two different ways: Either Scanning-TEM (STEM) is used to record a spectrum at each point of the image (STEMEELS/EDX mapping) or images are recorded at the several energy losses (ESI-Series). Traditionally STEM-EELS/EDX has been privileged [1], since the loss in resolution in STEM in comparison to TEM was less severe than the loss in energy resolution between EELS spectroscopy and ESI series. Lately it has been shown that ESI series are a valid alternative to EELS/EDX mapping in the core loss region, although data treatment to correct for the anisochromaticity is time consuming [2]. The situation changed with the introduction of highly corrected energy filters and monochromators (MC). Isochromaticity is greatly reduced during acquisition and the energy resolution of monochromated ESI series are show advantages over STEM EELS mapping without MC. All regions of the spectrum including the low-loss region can be explored by ESI series. The wealth of information contained in the low-loss energy range leading to valence, plasmon [3] and band-gap information can be now resolved with high spatial resolution. In this abstract we would like to demonstrate the capability of ESI series to resolve the chemical composition of nanoparticles.

Metallic (Au-Pd) nanoparticles on the order of $5 \mathrm{~nm}$ in diameter were shown to exhibit a 'halo' structure when viewed in filtered TEM mode at $5 \mathrm{eV}$ loss. This contrast likely arises due to surface plasmon resonance effects, which are shape- and composition dependent [4]. EFTEM spectrum imaging methods give access to spectroscopic data with sufficient energy and spatial resolution to interpret compositional heterogeneity within a single sub-5nm nanoparticle. It is important to note that this level of detail can be acquired rapidly without the use of STEM techniques. An image stack (Figure 1) was obtained at energies ranging from 5 $\mathrm{eV}$ to $81 \mathrm{eV}$ in steps of $4 \mathrm{eV}$ at a window width of $2 \mathrm{eV}$. It was shown spectroscopically (Figure 2) by the analysis of the $\mathrm{N}_{2,3}$ edge of $\mathrm{Pd}$ and the $\mathrm{O}_{2,3}$ edge of Au that the Au-Pd particle exhibited two compositionally discrete grains, one rich in $\mathrm{Au}$, the other in Pd.

Access to spectra and imaging at energy losses less than $10 \mathrm{eV}$ were made possible by implementing a monochromated source on the Zeiss Libra 200 CRISP located at the Caesar Research Institute in Bonn, Germany. Au/Pd nanoparticles were graciously provided by M. Yacaman of the University of Texas at San Antonio. 


\section{References}

[1] C. J. Kiely et al. Microsc Microanal 13(Suppl 2), 2007

[2] Werner Grogger et al, Journal of Electron Spectroscopy and Related Phenomena 143 (2005) 139-147

[3] J. Nelayah, Abstract EMC 2008

[4] A.S. Eggeman et al., Journal of Applied Physics 101 (2007) 024307

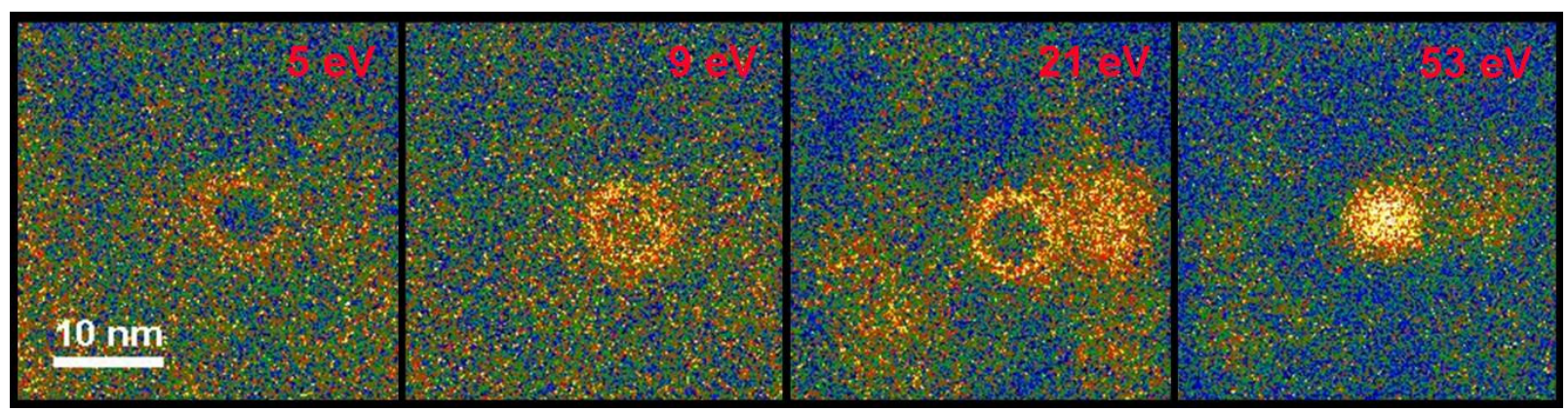

Figure 1: EFTEM images of an Au-Pd nanoparticle on carbon support film at 5, 9, 21 and 53 $\mathrm{eV}$, indicating surface and bulk plasmon resonance contrast.

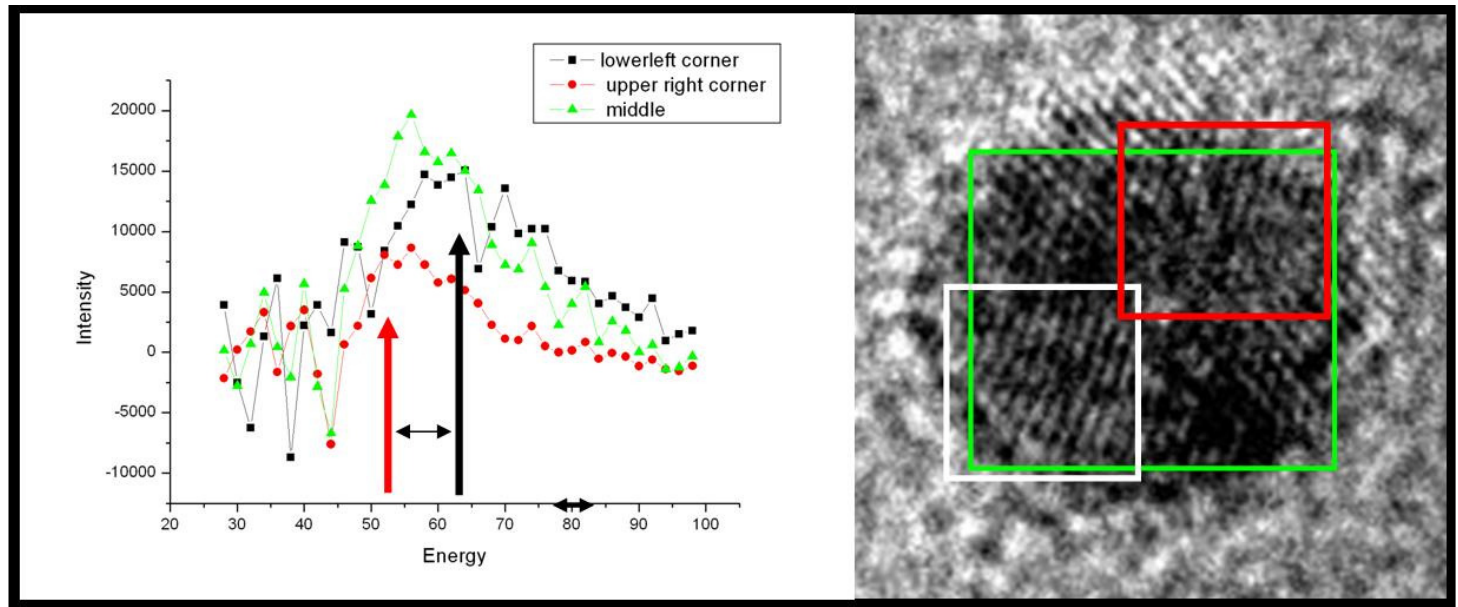

Figure 2: Analysis of an EFTEM spectrum image stack, indicating that the red and white regions differ compositionally, by comparing the $\mathrm{Au} \mathrm{O}_{2,3}$ edge with the $\mathrm{Pd} \mathrm{N}_{2,3}$ edge. 